\title{
Crystal structure of diethyl
}

\section{3,9-bis(4-fluorophenyl)-6,12-diphenyl-3,9- diazapentacyclo $\left[6 \cdot 4 \cdot 0.0^{2,7} \cdot 0^{4,11}\right]$ dodecane- 1,5-dicarboxylate, $\mathrm{C}_{40} \mathrm{H}_{36} \mathrm{~F}_{2} \mathrm{~N}_{2} \mathrm{O}_{4}$}

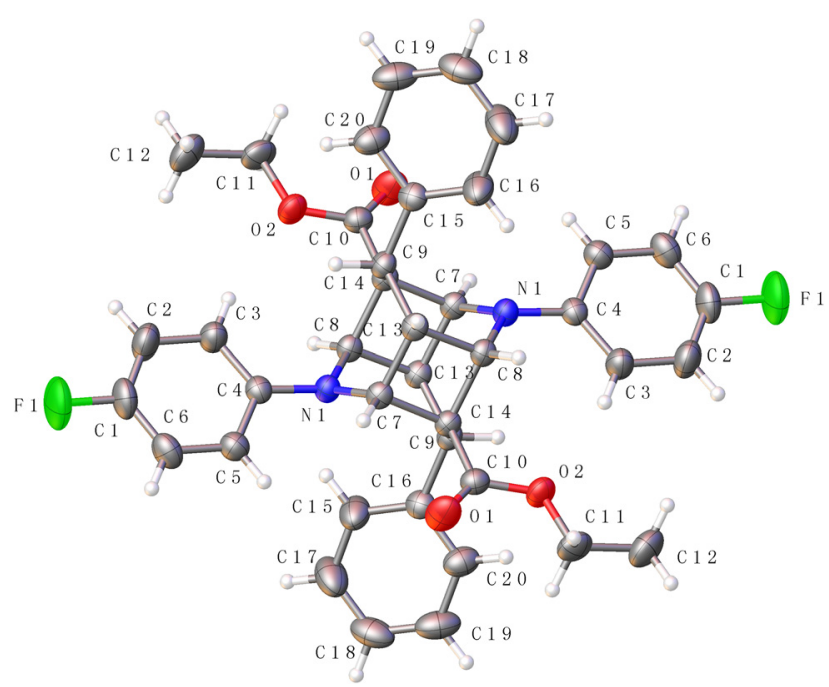

https://doi.org/10.1515/ncrs-2020-0552

Received October 26, 2020; accepted November 26, 2020; published online December 21, 2020

\section{Abstract \\ $\mathrm{C}_{40} \mathrm{H}_{36} \mathrm{~F}_{2} \mathrm{~N}_{2} \mathrm{O}_{4}$, monoclinic, $P 2_{1} / c$ (no. 14), $a=8.0755(7) \AA$, $b=19.7663(19) \AA, c=10.1987(9) \AA, \beta=95.940(2)^{\circ}$, $V=1619.2(3) \AA^{3}, Z=4, R_{\mathrm{gt}}(F)=0.0465, w R_{\mathrm{ref}}\left(F^{2}\right)=0.1188$, $\mathrm{T}=296 \mathrm{~K}$.}

\section{CCDC no.: 2040511}

The molecular structure is shown in the Figure. Table 1 contains crystallographic data and Table 2 contains the list of the atoms including atomic coordinates and displacement parameters.

*Corresponding author: Yue-Sheng Xie, School of Pharmacy, North China University of Science and Technology, 063210, Caofeidian District, Tangshan, P. R. China, E-mail: abcd.98@163.com. https:// orcid.org/0000-0002-6127-8861

Han-Qiao Shao, Yuan-Ming Lu and Qi-Wei Lin, School of Pharmacy, North China University of Science and Technology, 063210, Caofeidian District, Tangshan, P. R. China

Yu-Cai Wang, School of Continuing Education, JIA MU SI University, No. 148 Xuefu St., Jiamusi, Heilongjiang, P. R. China
Table 1: Data collection and handling.

\begin{tabular}{ll}
\hline Crystal: & Purple block \\
Size: & $0.25 \times 0.23 \times 0.20 \mathrm{~mm}$ \\
Wavelength: & Mo $K \alpha$ radiation $(0.71073 \AA)$ \\
$\mu:$ & $0.09 \mathrm{~mm}^{-1}$ \\
Diffractometer, scan mode: & Bruker APEX-II, $\varphi$ and $\omega$ \\
$\theta_{\text {max }}$, completeness: & $25.0^{\circ},>99 \%$ \\
$N\left(h k l_{\text {measured }}, N\left(h k l l_{\text {unique }}, R_{\text {int }}:\right.\right.$ & $8118,2856,0.018$ \\
Criterion for $I_{\text {obs }}, N(h k l)_{\text {gt }}:$ & $I_{\text {obs }}>2 \sigma\left(I_{\text {obs }}\right), 2380$ \\
$N(\text { param })_{\text {refined }}:$ & 218 \\
Programs: & Bruker [1], SHELX [2, 3] \\
\hline
\end{tabular}

\section{Source of material}

Preparation of 1,4-diaryl-1,4-dihydropyridine-3-carboxylic acid photoreactive raw materials was performed by a literature method [4]. Starting with 4-fluoroaniline $(0.5 \mathrm{mmol})$ ethyl propiolate $(0.5 \mathrm{mmol})$ and 4-fluorocinnamaldehyde (0.5 mmol) as starting materials; piperazine $(0.25 \mathrm{mmol})$ and $p$-toluenesulfonic $(0.02 \mathrm{mmol})$ acid were used as catalysts, and 1,2-dichloroethane $(20 \mathrm{~mL})$ was used as a solvent. The mixture was heated under reflux for $12 \mathrm{~h}$ to obtain 1,4(4-fluorodiaryl)-1,4-dihydrogen ethyl pyridine-3-carboxylate. The product of 1,4-(4-fluorodiaryl)-1,4-dihydrogen ethyl pyridine-3-carboxylate $(0.5 \mathrm{mmol})$ obtained in the first step of the reaction was placed on a blue LED (365 nm) for a photoreaction for $3 \mathrm{~h}$ [5]. The product was obtained by column chromatography eluting with petroleum ether and $n$-hexane 9:1. The resulting solution was evaporated to dryness to get some purple crystals.

\section{Experimental details}

All hydrogen atoms were placed in the calculated positions and all the non-hydrogen atoms were refined anisotropically. 
Table 2: Fractional atomic coordinates and isotropic or equivalent isotropic displacement parameters $\left(\AA^{2}\right)$.

\begin{tabular}{|c|c|c|c|c|}
\hline Atom & $x$ & $y$ & $z$ & $U_{\text {iso }} * / U_{\text {eq }}$ \\
\hline C1 & $0.2612(3)$ & 0.38980 (12) & $-0.03214(19)$ & $0.0506(5)$ \\
\hline$C 2$ & $0.2643(3)$ & $0.45774(12)$ & $-0.00638(19)$ & $0.0530(6)$ \\
\hline $\mathrm{H} 2$ & 0.219324 & 0.488426 & -0.069413 & $0.064^{\star}$ \\
\hline $\mathrm{C} 3$ & $0.3360(3)$ & $0.48006(10)$ & $0.11581(18)$ & $0.0422(5)$ \\
\hline H3 & 0.337826 & 0.526057 & 0.135123 & $0.051^{\star}$ \\
\hline $\mathrm{C} 4$ & $0.4052(2)$ & $0.43413(9)$ & $0.20949(16)$ & 0.0307 (4) \\
\hline $\mathrm{C} 5$ & $0.4004(2)$ & $0.36536(9)$ & $0.17786(18)$ & $0.0378(4)$ \\
\hline H5 & 0.446794 & 0.334099 & 0.239191 & $0.045^{\star}$ \\
\hline C6 & $0.3277(3)$ & $0.34296(11)$ & 0.05657 (19) & $0.0460(5)$ \\
\hline H6 & 0.324367 & 0.297099 & 0.036091 & $0.055^{\star}$ \\
\hline $\mathrm{C} 7$ & $0.4232(2)$ & $0.42666(8)$ & $0.45182(16)$ & $0.0288(4)$ \\
\hline $\mathrm{H} 7$ & 0.370654 & 0.382327 & 0.435837 & $0.035^{\star}$ \\
\hline $\mathrm{C} 8$ & $0.5549(2)$ & $0.52102(8)$ & $0.35474(16)$ & $0.0280(4)$ \\
\hline H8 & 0.595585 & 0.539625 & 0.274982 & $0.034^{\star}$ \\
\hline C9 & $0.4453(2)$ & $0.57404(8)$ & $0.42579(16)$ & $0.0282(4)$ \\
\hline C10 & $0.4319(2)$ & $0.64193(9)$ & $0.35726(18)$ & $0.0327(4)$ \\
\hline C11 & $0.3314(3)$ & $0.69737(10)$ & $0.1559(2)$ & $0.0482(5)$ \\
\hline $\mathrm{H} 11 \mathrm{~A}$ & 0.438531 & 0.716471 & 0.141242 & $0.058^{\star}$ \\
\hline $\mathrm{H} 11 \mathrm{~B}$ & 0.269044 & 0.730918 & 0.199922 & $0.058^{\star}$ \\
\hline C12 & $0.2377(3)$ & 0.67694 (13) & $0.0279(2)$ & $0.0602(6)$ \\
\hline $\mathrm{H} 12 \mathrm{~A}$ & 0.299568 & 0.642987 & -0.013490 & 0.090 * \\
\hline H12B & 0.222353 & 0.715676 & -0.028880 & $0.090^{\star}$ \\
\hline $\mathrm{H} 12 \mathrm{C}$ & 0.131075 & 0.659058 & 0.043757 & $0.090^{*}$ \\
\hline C13 & $0.6891(2)$ & $0.52386(9)$ & $0.47627(16)$ & $0.0291(4)$ \\
\hline H13 & 0.791449 & 0.545951 & 0.454644 & $0.035^{\star}$ \\
\hline C14 & $0.7257(2)$ & $0.45729(8)$ & $0.55052(16)$ & $0.0292(4)$ \\
\hline H14 & 0.780555 & 0.469473 & 0.637536 & $0.035^{\star}$ \\
\hline C15 & $0.8429(2)$ & $0.40882(9)$ & $0.48957(17)$ & $0.0326(4)$ \\
\hline C16 & $0.8627(3)$ & $0.40722(13)$ & $0.3565(2)$ & $0.0532(6)$ \\
\hline H16 & 0.801432 & 0.436618 & 0.299200 & $0.064^{\star}$ \\
\hline C17 & $0.9736(3)$ & $0.36201(14)$ & $0.3075(2)$ & $0.0635(7)$ \\
\hline H17 & 0.985820 & 0.361708 & 0.217783 & $0.076^{*}$ \\
\hline C18 & $1.0646(3)$ & $0.31811(12)$ & $0.3898(3)$ & $0.0572(6)$ \\
\hline H18 & 1.136681 & 0.287426 & 0.356236 & 0.069 * \\
\hline C19 & $1.0487(3)$ & $0.31978(11)$ & $0.5223(3)$ & $0.0562(6)$ \\
\hline H19 & 1.111280 & 0.290567 & 0.579180 & $0.067^{\star}$ \\
\hline C20 & $0.9397(2)$ & $0.36478(10)$ & $0.5714(2)$ & $0.0439(5)$ \\
\hline $\mathrm{H} 20$ & 0.930915 & 0.365630 & 0.661547 & $0.053^{*}$ \\
\hline $\mathrm{F} 1$ & $0.1925(2)$ & $0.36817(8)$ & $-0.15360(13)$ & $0.0834(5)$ \\
\hline N1 & $0.48461(17)$ & $0.45403(7)$ & $0.33375(13)$ & 0.0296 \\
\hline 01 & $0.4868(2)$ & $0.69432(7)$ & $0.40243(15)$ & $0.0570(4)$ \\
\hline 02 & 0.35359 (17) & $0.63626(6)$ & $0.23566(12)$ & 0.0407 (3) \\
\hline
\end{tabular}

\section{Comment}

Cage dimer 4-aryl-1,4-dihydropyridines have a wide range of biological activities, such as anti-HIV and as an antimultidrug resistance modulator [6-8]. Two caged dimeric compounds similar to the title compound have also been reported $[9,10]$. Dimerization to form cage compounds by photoreaction is an alternative to our previous synthetic protocol [11]. When the wavelength of the UV lamp is adjusted to $365 \mathrm{~nm}$, a complete [2 + 2] reaction occurs, and the title compound is completely cyclized (see picture). It is worth to note that compared to previous studies, it can be shown that the electronic effect has little or no effect on the cyclization, and the incident wavelength plays a decisive role in the cycloaddition reaction. In the crystal structure, several important bond angle data are involved: C4-N1$\mathrm{C} 8=121.38(13)^{\circ}, \mathrm{C} 9-\mathrm{C} 10-01=125.64(16)^{\circ}, \mathrm{C} 8-\mathrm{C} 13-\mathrm{C} 14=$ $116.39(13)^{\circ}, \mathrm{C} 8-\mathrm{N} 1-\mathrm{C} 13=112.31(13)^{\circ}$. The bond lengths and angles are in the expected ranges [9-11].

Author contribution: All the authors have accepted responsibility for the entire content of this submitted manuscript and approved submission.

Research funding: None declared.

Conflict of interest statement: The authors declare no conflicts of interest regarding this article.

\section{References}

1. BRUKER. SAINT. Version 8.23B; Bruker AXS Inc.: Madison, Wisconsin, USA, 2013.

2. Sheldrick G. M. Crystal structure refinement with SHELXL. Acta Crystallogr. 2015, C71, 3-8.

3. Sheldrick G. M. A short history of SHELX. Acta Crystallogr. 2008, A64, 112-122.

4. Wan J. P., Lin Y. F., Jing Y. F. Selectivity tunable divergent synthesis of 1,4-and 1,2-dihydropyridines via three-component reactions. Tetrahedron 2014, 70, 7874-7880.

5. Hilgeroth A., Baumeister U., Heinemann F. W. Solutiondimerization of 4-aryl-1,4-dihydropyridines. Eur. J. Org Chem. 2000, 2000, 245-249.

6. Hilgeroth A., Langner A. First bioanalytical evaluation of nonpeptidic cage dimeric HIV-1 protease inhibitor $N$-benzyl 4-aryl-1,4-dihydropyridine $\mathrm{H} 17$ : biotransformation and toxicity on Hep G2 cells. Arch. Pharm. 2000, 333, 32-34.

7. Hilgeroth A., Billich A. Cage dimeric 4-aryl-1,4-dihydropyridines as promising lead structures for the development of a novel class of HIV-1 protease inhibitors. Arch. Pharm. 1999, 332, 3-5.

8. Coburger C., Wollmann J., Krug M., Baumert C., Seifert M., Molnar J., Lage H., Hilgeroth A. Novel structure-activity relationships and selectivity profiling of cage dimeric 1,4-dihydropyridines as multidrug resistance (MDR) modulators. Bioorg. Med. Chem. 2010, 18, 4983-4990.

9. Zhu H., LiY. F., Guo Y. Y., Liu S., Lan H. Y., Zhuang P. Y., Zhong Q. D. Crystal structure of dimethyl 3,9-dibenzyl-6,12-dicyclohexyl-3,9diazahexacyclo $\left[6 \cdot 4 \cdot 0 \cdot 0^{2,7} \cdot 0^{4,11} \cdot 0^{5,10}\right]$-dodecane-1,11-dicarboxylate, $\mathrm{C}_{40} \mathrm{H}_{50} \mathrm{~N}_{2} \mathrm{O}_{4}$. Z. Kristallogr. NCS 2018, 233, 325-326.

10. ZhuH.,GuoY.Y.,LiuS., LiY.F., LanH.Y.,ZhuangP.Y.,ZhongQ.D.Crystal structure of diethyl 3,9-diphenyl-6,12-bis(3-methoxyphenyl)3,9-diazahexacyclo $\left[6 \cdot 4 \cdot 0 \cdot 0^{2,7} \cdot 0^{4,11} \cdot 0^{5,10}\right]$ dodecane1,11-dicarboxylate, $\mathrm{C}_{42} \mathrm{H}_{42} \mathrm{~N}_{2} \mathrm{O}_{6}$. Z. Kristallogr. NCS 2018, 233 , 327-328.

11. Guo Y. K., Chen S. J., Zhang M. M., Lan H. Y., Zhong Q. D. Z. Kristallogr. NCS 2019, 236, 425-427. 